TRANSACTIONS OF THE

AMERICAN MATHEMATICAL SOCIETY

Volume 355, Number 12, Pages 4825-4846

S 0002-9947(03)03117-9

Article electronically published on July 24, 2003

\title{
A GEOMETRIC CHARACTERIZATION OF VASSILIEV INVARIANTS
}

\author{
MICHAEL EISERMANN
}

\begin{abstract}
It is a well-known paradigm to consider Vassiliev invariants as polynomials on the set of knots. We prove the following characterization: a rational knot invariant is a Vassiliev invariant of degree $\leq m$ if and only if it is a polynomial of degree $\leq m$ on every geometric sequence of knots. Here a sequence $K_{z}$ with $z \in \mathbb{Z}$ is called geometric if the knots $K_{z}$ coincide outside a ball $B$, inside of which they satisfy $K_{z} \cap B=\tau^{z}$ for all $z$ and some pure braid $\tau$. As an application we show that the torsion in the braid group over the sphere induces torsion at the level of Vassiliev invariants: there exist knots in $\mathbb{S}^{1} \times \mathbb{S}^{2}$ that can be distinguished by $\mathbb{Z} / 2$-invariants of finite type but not by rational invariants of finite type. In order to obtain such torsion invariants we construct over $\mathbb{Z}$ a universal Vassiliev invariant of degree 1 for knots in $\mathbb{S}^{1} \times \mathbb{S}^{2}$.
\end{abstract}

\section{INTRODUCTION AND STATEMENT OF RESULTS}

A Vassiliev invariant is a map $v: \mathcal{K} \rightarrow A$ from the set of knots $\mathcal{K}$ to an abelian group $A$ such that $v$ satisfies a certain finiteness condition (see §1). Vassiliev invariants are commonly interpreted as polynomials on the set of knots [2, 3, 21]. One instance of this analogy is the following criterion:

Theorem (J. Dean [6], R. Trapp [20]). A Vassiliev invariant $v: \mathcal{K} \rightarrow \mathbb{Q}$ of degree $\leq m$ is a polynomial of degree $\leq m$ on every twist sequence of knots.

A twist sequence is a family of knots $K_{z}$ (indexed by $z \in \mathbb{Z}$ ) that are the same outside a ball, inside of which they differ as depicted in Figure 1 Using this criterion, J. Dean and R. Trapp showed that the class of Vassiliev invariants does not contain certain classical knot invariants such as crossing number, genus, signature, unknotting number, bridge number or braid index.

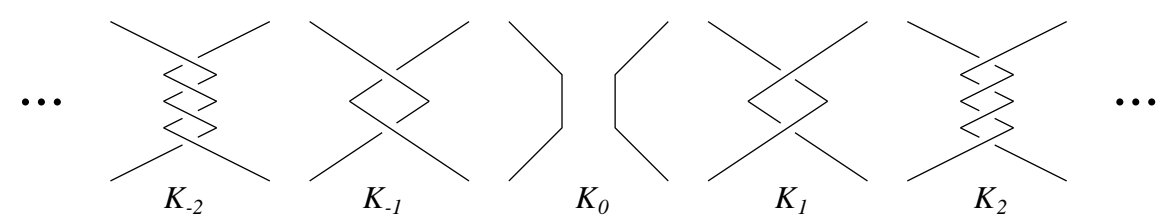

FiguRE 1. Local picture of a twist sequence of knots

Received by the editors March 5, 2001 and, in revised form, May 20, 2002.

2000 Mathematics Subject Classification. Primary 57M27, 57M25, 20F36.

Key words and phrases. Vassiliev invariant, invariant of finite type, twist sequence, geometric sequence of knots, torsion in the braid group over the sphere, Dirac twist, Dirac's spin trick. 
This article addresses the question of how to characterize Vassiliev invariants by means of such geometric conditions. To begin with, twist sequences alone do not suffice, as shown in $\$ 5$ by means of a counterexample.

Geometric characterization. Let $M$ be a 3 -manifold, and let $\mathcal{K} M$ be its set of knots considered up to isotopy. A geometric sequence of knots is a sequence $K_{z}$ indexed by $z \in \mathbb{Z}$ such that the knots $K_{z}$ coincide outside a ball $B \subset M$, inside of which they satisfy $K_{z} \cap B=\tau^{z}$ for all $z$ and some pure braid $\tau$. Such sequences were introduced by T. Stanford [19] under the name tangle maps.

We establish the following characterization of rational Vassiliev invariants.

Theorem 1 (proved in $₫ 4)$. A map $v: \mathcal{K} M \rightarrow \mathbb{Q}$ is a Vassiliev invariant of degree $\leq m$ if and only if it is a polynomial of degree $\leq m$ on every geometric sequence.

A twist sequence is a special case of a geometric sequence where $\tau$ is a full twist of only two strands, as shown in Figure 1 We explain in $\$ 5$ that twist sequences alone do not suffice to characterize Vassiliev invariants. For knots in the 3-sphere, however, we are led to the following characterization.

Theorem 2 (proved in $\oint_{6}$ ). A map $v: \mathcal{K S}^{3} \rightarrow \mathbb{Q}$ is a Vassiliev invariant of degree $\leq m$ if and only if $v$ is a polynomial on every twist sequence of knots and globally bounded by a polynomial of degree $m$ in the crossing number.

For a knot invariant with values in a torsion group the characterization is less simple: the geometric sequence condition of Theorem 1 is necessary but perhaps not sufficient, and the boundedness condition of Theorem 2 cannot even be formulated. In this case a characterization can still be established using geometric lattices instead of sequences (see $\$ 3$, Theorem 28).

The geometric characterization extends verbatim to Vassiliev invariants of links, tangles or embedded graphs. Generally speaking, these results give further evidence to the paradigm that Vassiliev invariants are polynomials.

Torsion invariants. Section 9 uses Dirac's spin trick to construct an involution $\theta$ on the set of knots in a reducible 3-manifold. Using geometric sequences, it is easy to see that rational Vassiliev invariants cannot distinguish a knot $K$ from its twin knot $\theta K$. For the manifold $\mathbb{S}^{1} \times \mathbb{S}^{2}$ we prove:

Theorem 3 (proved in $\S \$ 9$ [10). For every knot $K$ in $\mathbb{S}^{1} \times \mathbb{S}^{2}$ having homology class $[K] \in\{ \pm 3, \pm 5, \pm 7, \ldots\}$ the following assertions hold:

(1) The knot $K$ and its twin $\theta K$ are distinguished by a suitable Vassiliev invariant $\mathcal{K}\left(\mathbb{S}^{1} \times \mathbb{S}^{2}\right) \rightarrow \mathbb{Z} / 2$ of degree 1 .

(2) The knot $K$ and its twin $\theta K$ cannot be distinguished by any Vassiliev invariant $\mathcal{K}\left(\mathbb{S}^{1} \times \mathbb{S}^{2}\right) \rightarrow A$ if the abelian group $A$ has no 2 -torsion.

In particular, rational invariants of finite type do not distinguish all knots in $\mathbb{S}^{1} \times \mathbb{S}^{2}$.

Since this is the first occurrence of torsion in the Vassiliev theory of knots, we analyze this example in more detail. In Section[10 we carry out a combinatorial integration in order to construct a $\mathbb{Z}$-universal Vassiliev invariant of degree 1:

Theorem 4 (proved in $§ 10$. Let $M=\mathbb{S}^{1} \times \mathbb{S}^{2}$, and let $\mathcal{A}_{1} M$ be the $\mathbb{Z}$-module of chord diagrams modulo the obvious $1 T$ and Kirby relations (as explained in \$10.2). Then there exists a universal Vassiliev invariant $Z_{1}^{M}: \mathcal{K} M \rightarrow \mathcal{A}_{1} M$ of degree 1 . 
The $\mathbb{Z}$-module $\mathcal{A}_{1} M$ splits into a free part and a non-trivial torsion part, which turns out to be a vector space over $\mathbb{Z} / 2$ as explained in Proposition 60. The preceding theorem thus allows us to integrate the torsion invariants needed for Theorem 3.

To put this result in perspective, we remark that J. Lieberum [16] has constructed a Kontsevich isomorphism for knots in $\mathbb{S}^{1} \times \mathbb{S}^{2}$. Since the Kontsevich integral and its generalizations only work in characteristic zero, one generally loses the torsion part. Theorem 3 tells us that the 2-torsion contains essential information about knots in $\mathbb{S}^{1} \times \mathbb{S}^{2}$. It is still an open question whether this can occur for knots in the 3 -sphere.

How this paper is organized. Section 1 recapitulates the combinatorial definition of Vassiliev invariants, while Section 2 briefly recalls the definition of discrete polynomial functions. They are combined in Section 3 to characterize Vassiliev invariants via geometric lattices. Section 4 reduces this characterization to geometric sequences. As an extended example, Section 5 discusses the determinant of knots and shows that twist sequences alone do not suffice to characterize Vassiliev invariants, while Section 6 explains that the crucial condition is polynomial growth.

The second part of this paper deals with torsion. Section 7 examines the polynomial criterion for Vassiliev invariants with values in a torsion group. Section 8 shows that the torsion in the braid group over the sphere induces torsion at the level of Vassiliev invariants. This is applied to knots in $\mathbb{S}^{1} \times \mathbb{S}^{2}$ in Section 9 The final Section 10 analyzes the torsion at the level of chord diagrams of knots in $\mathbb{S}^{1} \times \mathbb{S}^{2}$ and constructs over $\mathbb{Z}$ a universal Vassiliev invariant of degree 1.

\section{VASSILIEV INVARIANTS}

We first recall the axiomatic definition of Vassiliev invariants via singular knots, as it was formulated by J.S. Birman and X.-S. Lin [5]. We will also make use of the dual definition via the Vassiliev filtration of knots.

1.1. Vassiliev invariants. Let $M$ be a 3-manifold. A knot is a smooth embedding $\mathbb{S}^{1} \hookrightarrow M$, regarded up to isotopy. Each knot is oriented by the standard orientation of $\mathbb{S}^{1}$. A singular knot is an immersion $\mathbb{S}^{1} \rightarrow M$ such that each multiple point is a double point according to the local model $\chi$.

Let $\mathcal{K}$ be the set of knots in $M$ and $\mathcal{K}_{n}$ the set of singular knots with $n$ double points. Assuming that $M$ is oriented, every knot invariant $v: \mathcal{K} \rightarrow A$ with values in an abelian group $A$ can be uniquely extended to a family of maps $v^{(n)}: \mathcal{K}_{n} \rightarrow A$ by the so-called Vassiliev skein relation

$$
v^{(n)}(\searrow)=v^{(n-1)}(\nwarrow)-v^{(n-1)}(\searrow)
$$

with the initial condition $v^{(0)}=v$. This recursive formula is to be interpreted as a local resolution of the depicted singularity, and the orientation of $M$ determines the sign of the resolved knots $\measuredangle$ and $\searrow$. Evidently the definition of $v^{(n)}$ is independent of the order in which the singularities are resolved.

Definition 5. A knot invariant $v: \mathcal{K} \rightarrow A$ is a Vassiliev invariant of degree $\leq m$ if $v^{(m+1)}$ vanishes. In this case $v$ is also called an invariant of type $\leq m$ or simply an invariant of finite type. 
The map $v^{(n)}$ can be regarded as the $n$-th derivative of $v$ (see Remark 27). The vanishing of $v^{(m+1)}$ then means that $v$ is a polynomial of degree $\leq m$. The aim of Sections 2 and 3 is to turn this analogy into a characterization.

Remark 6. Many important knot invariants are of finite type, most notably the coefficients of the Jones polynomial [5] (after a suitable change of variable) and its generalizations, the so-called quantum invariants of knots [2].

1.2. The Vassiliev filtration. There is a dual version of Definition 5, which will be useful in the sequel. Let $\mathbb{Z} \mathcal{K}$ be the free $\mathbb{Z}$-module with basis $\mathcal{K}$, and let $\partial^{n}$ : $\mathbb{Z} \mathcal{K}_{n} \rightarrow \mathbb{Z} \mathcal{K}$ be the map that is given by resolution of singularities following the pattern $\chi \mapsto \measuredangle-\chi$. This means that each $n$-singular knot is mapped to an alternating sum of $2^{n}$ non-singular knots.

Remark 7. Every knot invariant $v: \mathcal{K} \rightarrow A$ extends to a linear map $\mathbb{Z} \mathcal{K} \rightarrow A$, which we also denote by $v$. By definition of $\partial^{n}$ we have $v^{(n)}=v \circ \partial^{n}$. This means that $v$ is of degree $\leq m$ if and only if it vanishes on $\operatorname{im}\left(\partial^{m+1}\right)$.

Definition 8. We set $\mathcal{F}_{n}=\operatorname{im}\left(\partial^{n}: \mathbb{Z} \mathcal{K}_{n} \rightarrow \mathbb{Z} \mathcal{K}\right)$. The sequence of submodules $\mathbb{Z} \mathcal{K}=\mathcal{F}_{0} \supset \mathcal{F}_{1} \supset \mathcal{F}_{2} \supset \cdots$ is called the Vassiliev filtration.

Remark 9 (Orientability). The Vassiliev filtration does not require that $M$ is orientable: a local orientation around each singularity suffices to define $\partial^{n}$, and the choice of orientations affects only the sign. The module $\operatorname{im}\left(\partial^{n}\right)$ is thus well-defined. This allows us to extend the notion of Vassiliev invariants to knots in a non-orientable manifold.

Remark 10 (Knotted objects). The notion of Vassiliev filtration is based on the idea of resolving singularities, i.e. self-intersections of a 1-dimensional object in a 3 -dimensional ambient space. The definition immediately extends to braids, links, tangles, embedded graphs, and other classes of knotted objects.

\section{Discrete POLYNOMial FunCtions}

In order to fix our notation, let us briefly recall the definition of discrete polynomial functions $f: \mathbb{Z}^{d} \rightarrow A$ with values in an abelian group $A$. The discrete derivative $\partial_{i} f: \mathbb{Z}^{d} \rightarrow A$ is defined by $\left(\partial_{i} f\right)(z)=f\left(z+e_{i}\right)-f(z)$, where $e_{i}$ is the $i$-th vector in the standard basis of $\mathbb{Z}^{d}$. Obviously $\partial_{i} \partial_{j} f=\partial_{j} \partial_{i} f$. For a multi-index $\alpha \in \mathbb{N}^{d}$ we define $\partial^{\alpha}:=\partial_{1}^{\alpha_{1}} \cdots \partial_{d}^{\alpha_{d}}$. The sum $|\alpha|=\alpha_{1}+\cdots+\alpha_{d}$ is called the degree of the multi-index $\alpha$.

Definition 11. A map $f: \mathbb{Z}^{d} \rightarrow A$ is a polynomial of degree $\leq m$ if the discrete derivative $\partial^{\alpha} f$ vanishes for every multi-index $\alpha$ of degree $>m$.

Example 12. For $\alpha \in \mathbb{N}$ let $b^{\alpha}: \mathbb{Z} \rightarrow \mathbb{Z}$ be the binomial coefficient function

$$
b^{\alpha}(z)=\left(\begin{array}{c}
z \\
\alpha
\end{array}\right):=\frac{z(z-1) \cdots(z-\alpha+1)}{\alpha(\alpha-1) \cdots 1} .
$$

This includes the special case $\left(\begin{array}{l}z \\ 0\end{array}\right)=1$ and is extended to $\alpha<0$ by setting $\left(\begin{array}{l}z \\ \alpha\end{array}\right)=0$. More generally, for $\alpha \in \mathbb{N}^{d}$, let $b^{\alpha}: \mathbb{Z}^{d} \rightarrow \mathbb{Z}$ be given by $b^{\alpha}(z)=\left(\begin{array}{c}z \\ \alpha\end{array}\right):=\left(\begin{array}{c}z_{1} \\ \alpha_{1}\end{array}\right) \cdots\left(\begin{array}{c}z_{d} \\ \alpha_{d}\end{array}\right)$. We have $\partial^{\beta} b^{\alpha}=b^{\alpha-\beta}$; hence $b^{\alpha}$ is a polynomial function of degree $|\alpha|$.

Proposition 13. A map $f: \mathbb{Z}^{d} \rightarrow A$ is a polynomial of degree $\leq m$ if and only if it can be written as $f(z)=\sum_{|\alpha| \leq m} c_{\alpha} b^{\alpha}(z)$ with coefficients $c_{\alpha} \in A$. In this case the coefficients are uniquely determined by the formula $c_{\alpha}=\left(\partial^{\alpha} f\right)(0)$. 
Remark 14 . If $A$ is a vector space over $\mathbb{Q}$, then every polynomial $f: \mathbb{Z}^{d} \rightarrow A$ can be rewritten as $f(z)=\sum c_{\alpha}^{\prime} z^{\alpha}$ with coefficients $c_{\alpha}^{\prime} \in A$. In characteristic zero we thus recover the usual definition: a map $f: \mathbb{Z}^{d} \rightarrow \mathbb{Q}$ is a polynomial of degree $\leq m$ if and only if it is the restriction of a polynomial $\tilde{f}: \mathbb{Q}^{d} \rightarrow \mathbb{Q}$ of degree $\leq m$.

\section{Characterization of Vassiliev invariants via Geometric lattices}

This section discusses geometric lattices of braids and knots, and Theorem 28 characterizes Vassiliev invariants as polynomials on geometric lattices.

3.1. Geometric lattices of braids. Let $B_{n}$ be the group of braids on $n$ strands. It is generated by the elementary braids $\sigma_{1}, \sigma_{2}, \ldots, \sigma_{n-1}$, where $\sigma_{i}$ performs a positive half twist interchanging strands $i$ and $i+1$. The permutation of strands defines an epimorphism $\pi: B_{n} \rightarrow \Sigma_{n}$ onto the symmetric group on $n$ points. The kernel $P_{n}=\operatorname{ker}(\pi)$ is the group of pure braids.

For a pure braid $\tau$ we call $\tau^{z}$ with $z \in \mathbb{Z}$ a geometric sequence of braids, in analogy with geometric sequences of numbers. More generally, we have

Definition 15. Let $\beta_{0}, \beta_{1}, \ldots, \beta_{d} \in B_{n}$ be braids and let $\tau_{1}, \ldots, \tau_{d} \in P_{n}$ be pure braids. We call $\Phi: \mathbb{Z}^{d} \rightarrow B_{n}$ given by $\Phi(z)=\beta_{0} \tau_{1}^{z_{1}} \beta_{1} \cdots \tau_{d}^{z_{d}} \beta_{d}$ a $d$-dimensional geometric lattice of braids.

Lemma 16 (Polynomial criterion). Let $v: B_{n} \rightarrow A$ be a Vassiliev invariant of degree $\leq m$. Then for every geometric lattice $\Phi: \mathbb{Z}^{d} \rightarrow B_{n}$, the composition $v \circ \Phi: \mathbb{Z}^{d} \rightarrow A$ is a polynomial of degree $\leq m$.

Proof. One can define singular braids and Vassiliev invariants of braids as in $\$ 1.1$ The dual point of view of $\$ 1.2$, however, seems to be more natural in this setting: Let $I$ be the kernel of the epimorphism $\mathbb{Z} B_{n} \rightarrow \mathbb{Z} \Sigma_{n}$. The ideal $I$ is generated by the differences $\sigma_{i}^{+}-\sigma_{i}^{-}$, and the $I$-adic filtration $\mathbb{Z} B_{n} \supset I \supset I^{2} \supset \cdots$ is exactly the Vassiliev filtration of braids. By hypothesis, $v$ vanishes on $I^{m+1}$.

Let $\Phi(z)=\beta_{0} \tau_{1}^{z_{1}} \beta_{1} \cdots \tau_{d}^{z_{d}} \beta_{d}$ be a geometric lattice of braids, which we view as a map $\Phi: \mathbb{Z}^{d} \rightarrow \mathbb{Z} B_{n}$. To this map we can apply the difference operators $\partial_{1}, \ldots, \partial_{d}$ as explained in the previous section. For $\alpha \in \mathbb{N}^{d}$ we get

$$
\partial^{\alpha} \Phi(z)=\beta_{0} \tau_{1}^{z_{1}}\left(\tau_{1}-1\right)^{\alpha_{1}} \beta_{1} \cdots \tau_{d}^{z_{d}}\left(\tau_{d}-1\right)^{\alpha_{d}} \beta_{d},
$$

which is an element of $I^{|\alpha|}$. Composing this with our invariant $v$, we see that $\partial^{\alpha}(v \circ \Phi)=0$ for every multi-index $\alpha \in \mathbb{N}^{d}$ of degree $>m$. We conclude that $v \circ \Phi: \mathbb{Z}^{d} \rightarrow A$ is a polynomial of degree $\leq m$.

Remark 17 (Polynomial characterization). It is easy to see that the converse of Lemma [16 also holds. This means that Vassiliev invariants are characterized by their behaviour on geometric lattices. We will prove this statement for knots in Theorem 28 below.

Remark 18 (Tangles). Instead of pure braids one can equally well work with geometric sequences of pure tangles [19]. In this setting the braid groups $B_{n}$ and their Vassiliev filtration $\mathbb{Z} B_{n} \supset I \supset I^{2} \supset \cdots$ are replaced with the tangle category $\mathcal{T}$ and its Vassiliev filtration $\mathbb{Z} \mathcal{T} \supset J \supset J^{2} \supset \cdots$, where $J=(\swarrow-\chi)$ is the ideal generated by crossing changes. 
3.2. Geometric lattices of knots. In the sequel we consider a 3-manifold $M$ and the set $\mathcal{K}=\mathcal{K} M$ of knots in $M$.

Definition 19. A map $K: \mathbb{Z}^{d} \rightarrow \mathcal{K}$ is called a geometric lattice if the knots $K(z)$ coincide outside a ball $B \subset M$, inside of which the tangles $\Phi(z)=K(z) \cap B$ form a geometric lattice of braids. (Here and in the sequel we tacitly assume that each knot $K(z)$ intersects $\partial B$ transversely.)

Example 20 (Closed braids). For a braid $\beta \in B_{n}$ let $\operatorname{clos}(\beta)$ be its closure, i.e. the link in $\mathbb{S}^{3}$ obtained by identifying corresponding endpoints of the braid $\beta$. Every geometric lattice of braids $\Phi: \mathbb{Z}^{d} \rightarrow B_{n}$ defines a geometric lattice of links $K(z)=\operatorname{clos}(\Phi(z))$. If some $K(z)$ is a knot, then so are all members of the lattice.

Remark 21 (Inverse problem). Given an arbitrary family of knots, it may be quite hard to decide whether or not they can be arranged to form a geometric lattice. We will not address this "inverse problem", but explicitly construct all geometric lattices needed in the course of this paper.

We introduce geometric lattices $\mathbb{Z}^{d} \rightarrow \mathcal{K}$ of arbitrary dimension $d$ mainly for technical reasons. We will mostly be interested in geometric sequences of knots, i.e. in the case $d=1$. A typical example is the well-known family of torus knots:

Example 22 (Torus knots). For coprime integers $p, q$ with $p \geq 1$, the torus knot $T(p, q)$ is the closure of the braid $\left(\sigma_{1} \sigma_{2} \cdots \sigma_{p-1}\right)^{q} \in B_{p}$. The map $K: \mathbb{Z} \rightarrow \mathcal{K S}^{3}$ with $K(z)=T(p, q+z p)$ is a geometric sequence of torus knots.

3.3. Rearranging a geometric lattice. There are alternative ways to define a geometric lattice of knots. We will briefly explain that they are equivalent.

Proposition 23. A map $K: \mathbb{Z}^{d} \rightarrow \mathcal{K}$ is a geometric lattice if and only if there exist disjoint balls $B_{1}, \ldots, B_{d}$ and pure braids $\tau_{1}, \ldots, \tau_{d}$ such that the knots $K(z)$ coincide outside $B_{1} \cup \cdots \cup B_{d}$, and for each $i$ the tangle $K(z) \cap B_{i}$ is given by $\tau_{i}^{z_{i}}$.

Proof. If $K: \mathbb{Z}^{d} \rightarrow \mathcal{K}$ is a geometric lattice, then it obviously has the property stated in the proposition. This means that $K$ can be presented as in Figure2 below.

Conversely, assume that $K: \mathbb{Z}^{d} \rightarrow \mathcal{K}$ can be presented as in Figure 2 By an isotopy of $M$ we can place $B_{1}, \ldots, B_{d}$ side by side in a slightly bigger ball $B$ containing $B_{1} \cup \cdots \cup B_{d}$. In this situation we can define pure braids $\tilde{\tau}_{1}, \ldots, \tilde{\tau}_{d}$ in the obvious way to obtain $K(z) \cap B=\tilde{\tau}_{1}^{z_{1}} \cdots \tilde{\tau}_{d}^{z_{d}}$, as desired.
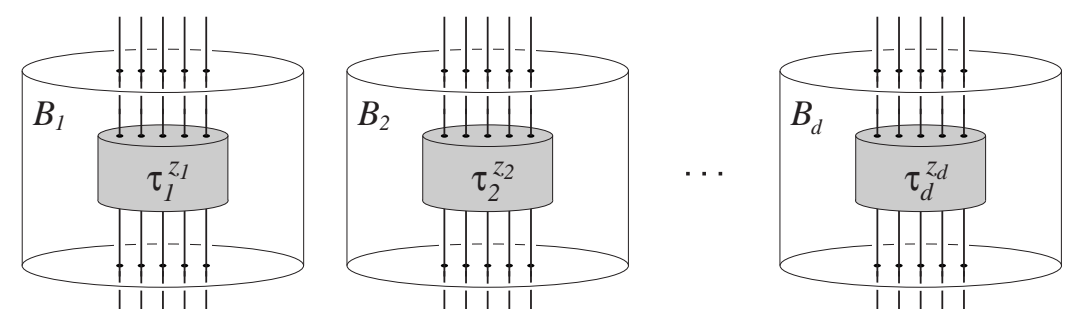

FIgURE 2. Alternative view of a geometric lattice $K\left(z_{1}, z_{2}, \ldots, z_{d}\right)$ 
Corollary 24 (Commuting braids). A map $K: \mathbb{Z}^{d} \rightarrow \mathcal{K}$ is a geometric lattice if and only if there exist a ball $B$ and commuting pure braids $\tilde{\tau}_{1}, \ldots, \tilde{\tau}_{d}$ such that the knots $K(z)$ coincide outside $B$ and the tangle $K(z) \cap B$ is given by $\tilde{\tau}_{1}^{z_{1}} \cdots \tilde{\tau}_{d}^{z_{d}}$.

Corollary 25 (Change of variables). If $K: \mathbb{Z}^{d} \rightarrow \mathcal{K}$ is a geometric lattice and $\varphi: \mathbb{Z}^{e} \rightarrow \mathbb{Z}^{d}$ is an affine map, then $K \circ \varphi: \mathbb{Z}^{e} \rightarrow \mathcal{K}$ is also a geometric lattice.

Please note that these arguments result from uniformly rearranging the family of knots $K(z)$ in $M$. This works fine for knots but not for braids; the assertions of Corollaries 24 and 25$]$ would be false for braids.

3.4. Twist lattices. As a special case of geometric lattice we have the following: A twist lattice $K: \mathbb{Z}^{d} \rightarrow \mathcal{K}$ is a family of knots as depicted in Figure 2, such that each pure braid $\tau_{i}$ is a full twist of only two strands. In the one-dimensional case $d=1$ we obtain a twist sequence, as explained in the introduction (see Figure 1).

Example 26 (Pretzel knots). For odd integers $t_{1}, \ldots, t_{d} \in \mathbb{Z}$ let $P\left(t_{1}, \ldots, t_{d}\right)$ be the pretzel link as depicted in Figure 3. If $d$ is odd, then this defines a twist lattice of knots, more precisely, $K: \mathbb{Z}^{d} \rightarrow \mathcal{K}$ given by $K\left(z_{1}, \ldots, z_{d}\right)=P\left(2 z_{1}-1, \ldots, 2 z_{d}-1\right)$ is a twist lattice of pretzel knots.

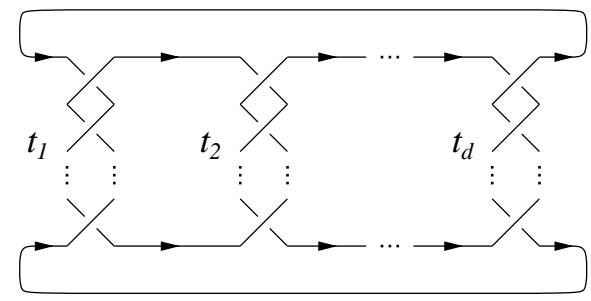

Figure 3 . The pretzel link $P\left(t_{1}, t_{2}, \ldots, t_{d}\right)$

Remark 27 (Twist lattice associated with a singular knot). Every knot $K \bullet$ with $n$ singularities defines a twist lattice $K: \mathbb{Z}^{n} \rightarrow \mathcal{K}$ by replacing each of its singular points $i=1, \ldots, n$ by a twist sequence $\sigma^{2 z_{i}-1}$. If we consider this as a map $K: \mathbb{Z}^{n} \rightarrow \mathbb{Z} \mathcal{K}$, then the derivative $\partial_{1} \cdots \partial_{n} K(0)$ is the same as the resolution $\partial^{n} K_{\bullet}$ introduced in $\$ 1.2$. Thus, given a knot invariant $v: \mathcal{K} \rightarrow A$, the composition $v \circ K: \mathbb{Z}^{n} \rightarrow A$ has derivative $\partial_{1} \cdots \partial_{n}(v \circ K)(0)=v^{(n)}\left(K_{\bullet}\right)$. This explains the interpretation of $v^{(n)}$ as an $n$-th derivative of the invariant $v$.

3.5. Characterization of Vassiliev invariants. The notion of geometric lattice being in place, it is an easy matter to prove that Vassiliev invariants are characterized by their behaviour on geometric lattices. The point is to give a precise meaning to the phrase "Vassiliev invariants are polynomials on the set of knots".

Theorem 28. Let $M$ be a 3-manifold and let $v: \mathcal{K} M \rightarrow A$ be a knot invariant with values in an abelian group $A$. The following conditions are equivalent:

(1) $v$ is a Vassiliev invariant of degree $\leq m$.

(2) $v$ is a polynomial of degree $\leq m$ of every geometric lattice of knots.

(3) $v$ is a polynomial of degree $\leq m$ of every twist lattice of knots. 
Proof. Implication $(1 \Rightarrow 2)$ follows from the proof of Lemma 16. More explicitly, let $K: \mathbb{Z}^{d} \rightarrow \mathcal{K}$ be a geometric lattice of knots. For every multi-index $\alpha \in \mathbb{N}^{d}$ of degree $|\alpha|=n$ the discrete derivative $\partial^{\alpha} K: \mathbb{Z}^{d} \rightarrow \mathbb{Z} \mathcal{K}$ takes values in $\mathcal{F}_{n}$. If $v: \mathcal{K} \rightarrow A$ is a Vassiliev invariant of degree $\leq m$, then $\partial^{\alpha}(v \circ K)$ vanishes for $|\alpha|>m$, and hence $v \circ K$ is a polynomial of degree $\leq m$, as claimed.

Implication $(2 \Rightarrow 3)$ is a specialization. To prove $(3 \Rightarrow 1)$, let $v: \mathcal{K} \rightarrow A$ be a knot invariant satisfying condition (3) and let $K_{\bullet}$ be an $n$-singular knot. We have to show that $v\left(K_{\bullet}\right)=0$ whenever $n>m$. The $n$-singular knot $K_{\bullet}$ defines a twist lattice $K: \mathbb{Z}^{n} \rightarrow \mathcal{K}$ as explained in the preceding remark. By hypothesis, the composition $v \circ K: \mathbb{Z}^{n} \rightarrow A$ is a polynomial of degree $\leq m$. Thus $v\left(K_{\bullet}\right)=\partial_{1} \cdots \partial_{n}(v \circ K)(0)=0$ whenever $n>m$.

\section{Characterization of Vassiliev invariants via geometric sequences}

Since sequences are a special case of lattices, we know that a Vassiliev invariant $v: \mathcal{K} \rightarrow \mathbb{Q}$ is a polynomial on every geometric sequence of knots. Quite surprisingly the converse is also true: rational Vassiliev invariants are characterized by their behaviour on geometric sequences alone. In order to prove this characterization, we first need a little lemma about polynomials.

4.1. Polynomial functions into torsion-free groups. The polynomial condition for $f: \mathbb{Z}^{d} \rightarrow A$ uses all directions $\partial_{i}$ in all possible combinations. In the torsion free case, however, it suffices to require the polynomial condition on straight lines, i.e. for every map $g: \mathbb{Z} \rightarrow \mathbb{Z}^{d}$ with $g(x)=a+x b$ and constants $a, b \in \mathbb{Z}^{d}$. This reduces the $d$-dimensional condition to a one-dimensional condition:

Lemma 29 (Straight lines). Let $A$ be an abelian group without torsion. A map $f: \mathbb{Z}^{d} \rightarrow A$ is a polynomial of degree $\leq m$ if and only if it is a polynomial of degree $\leq m$ on every straight line $g: \mathbb{Z} \rightarrow \mathbb{Z}^{d}$.

Proof. Since $A \rightarrow A \otimes \mathbb{Q}$ is injective, we can assume that $A$ is a vector space over $\mathbb{Q}$. Every polynomial $f: \mathbb{Z}^{d} \rightarrow A$ of degree $\leq m$ can then be written as $f(z)=\sum_{|\alpha| \leq m} c_{\alpha} z^{\alpha}$ with constants $c_{\alpha} \in A$, see Remark14, Note that such a presentation is in general not available if $A$ has torsion.

To prove " $\Rightarrow$ ", assume that $g: \mathbb{Z} \rightarrow \mathbb{Z}^{d}$ is a straight line. Then $f \circ g: \mathbb{Z} \rightarrow A$ is given by $f(g(x))=\sum_{0 \leq k \leq m} c_{k}^{\prime} x^{k}$, which is a polynomial of degree $\leq m$.

To prove " $\Leftarrow$ ", assume that $f$ is a polynomial of degree $\leq m$ on every straight line in $\mathbb{Z}^{d}$. In particular, this is true for $g(x)=a+x e_{i}$, which shows that $\partial_{i}^{m+1} f$ vanishes for every $i$. Therefore $f$ is a polynomial of degree $\leq d m$. It remains to be shown that the degree is at most $m$.

Let $n$ be the degree of $f$ and $f(z)=\sum_{|\alpha| \leq n} c_{\alpha} z^{\alpha}$. Let $f_{i}(z)=\sum_{|\alpha|=i} c_{\alpha} z^{\alpha}$ be the homogeneous part of degree $i$. We choose a point $b \in \mathbb{Z}^{d}$ such that $f_{n}(b) \neq 0$. On the straight line $g(x)=x b$ the polynomial $f$ restricts to $f(x b)=\sum_{i=0}^{n} x^{i} f_{i}(b)$, which is a polynomial of degree $n$. Using the hypothesis, we conclude $n \leq m$.

4.2. Characterization of Vassiliev invariants. We are now in position to prove the following one-dimensional characterization:

Theorem 30. Let $A$ be an abelian group without torsion. Then $v: \mathcal{K} \rightarrow A$ is a Vassiliev invariant of degree $\leq m$ if and only if $v$ is a polynomial of degree $\leq m$ on every geometric sequence of knots. 
Proof. The implication " $\Rightarrow$ " is a special case of Theorem 28 To prove " $\Leftarrow$ ", suppose that $\Phi: \mathbb{Z}^{d} \rightarrow \mathcal{K}$ is a geometric lattice of knots. Then every $\varphi: \mathbb{Z} \rightarrow \mathcal{K}$ with $\varphi(x)=$ $\Phi(a+x b)$ is a geometric sequence by Corollary 25. By hypothesis, $v \circ \varphi: \mathbb{Z} \rightarrow A$ is a polynomial of degree $\leq m$. Since $A$ is torsion-free, Lemma 29 ensures that $v \circ \Phi: \mathbb{Z}^{d} \rightarrow A$ is a polynomial of degree $\leq m$. By Theorem 28 we conclude that $v$ is a Vassiliev invariant of degree $\leq m$.

Question 31. Is this characterization valid for every abelian group?

If $A$ is a finite abelian group, then Lemma 29 and the argument of the previous proof are no longer valid (see Example 45 below). I could find neither an alternative proof nor a counterexample in the torsion case.

\section{THE DETERMINANT OF KNOTS}

Twist sequences are a special case of geometric sequences. The latter characterize Vassiliev invariants, but the former do not. As an example we analyze the determinant of knots: its square is "almost" a Vassiliev invariant in the sense that it is a polynomial on every twist lattice, yet it is not of finite type.

5.1. The determinant on twist sequences. Given a knot $K$ in $\mathbb{S}^{3}$, one can construct a Seifert surface spanning it and derive the associated Seifert matrix $V$ (see for example [15], Chapter 6). The symmetrized matrix $V+V^{\dagger}$ defines the quadratic form of the knot, up to a certain equivalence relation. Its determinant is a classical knot invariant and appears in various guises: for example, the absolute value $\left|\operatorname{det}\left(V+V^{\dagger}\right)\right|$ is the order of the first homology group $H_{1}(M)$, where $M$ is the two-fold cover of $\mathbb{S}^{3}$ branched along $K$.

Up to a sign the determinant coincides with the Alexander polynomial

$$
\Delta(K)=\operatorname{det}\left(t^{-\frac{1}{2}} V-t^{\frac{1}{2}} V^{\dagger}\right)
$$

evaluated at $t=-1$. For our purposes it will be most convenient to use the skein theoretic approach via the Conway polynomial (see [15], Chapter 8): the map $\nabla: \mathcal{L} \rightarrow \mathbb{Z}[z]$ is the unique link invariant that takes the value $\nabla(\bigcirc)=1$ on the unknot and satisfies the skein relation

$$
\nabla(\searrow)-\nabla(\searrow)=z \nabla(\rangle)
$$

The Alexander polynomial $\Delta(L)$ is obtained from $\nabla(L)$ by substituting $z=t^{\frac{1}{2}}-t^{-\frac{1}{2}}$. We thus define the (signed) determinant det $: \mathcal{L} \rightarrow \mathbb{Z}[2 i]$ by $\operatorname{det}(L)=\left.\nabla(L)\right|_{z=2 i}$. It satisfies the skein relation

$$
\operatorname{det}(\nwarrow)-\operatorname{det}(\searrow)=2 i \operatorname{det}(\rangle)
$$

which shows, by the way, that the determinant coincides with the value of the Jones polynomial at $t=-1$. This particular value has the remarkable property that, up to a sign, it is linear on every twist sequence:

Proposition 32. The squared determinant $\operatorname{det}^{2}: \mathcal{K S}^{3} \rightarrow \mathbb{Z}$ is a polynomial of degree $\leq 2$ on every twist sequence. It is hence a polynomial on every twist lattice.

Proof. If $K(n)$ is a twist sequence of knots, then the skein relation for the determinant translates, after a small calculation, into a simple recursive formula:

$$
\operatorname{det} K(n)-2 \varepsilon \operatorname{det} K(n-1)+\operatorname{det} K(n-2)=0,
$$


where $\varepsilon=-1$ if the twisted strands are parallel and $\varepsilon=1$ if the strands are anti-parallel. Given the values $a_{0}:=\operatorname{det} K(0)$ and $a_{1}:=\operatorname{det} K(1)$, we obtain

$$
\operatorname{det} K(n)=\varepsilon^{n} \cdot\left[a_{0}+n\left(\varepsilon a_{1}-a_{0}\right)\right] .
$$

In either case its square is constant or quadratic in $n$. This proves that $\operatorname{det}^{2}$ is a polynomial of degree $\leq 2$ on every twist sequence.

We remark that the determinant of a knot is an integer in $1+4 \mathbb{Z}$, which implies that the sign of $\operatorname{det}(K)$ can be recovered from $|\operatorname{det}(K)|$. In other words, the knot invariants det and $\operatorname{det}^{2}$ contain exactly the same information.

In order to illustrate the preceding characterization theorems, we give two proofs that $\operatorname{det}^{2}$ is not of finite type, the first using twist lattices (as in Theorem 28), the second using geometric sequences (as in Theorem 30).

5.2. The determinant on pretzel knots. The squared determinant is a polynomial of degree $\leq 2$ on every twist sequence and hence of degree $\leq 2 d$ on every $d$-dimensional twist lattice. The following calculation for the family of pretzel knots shows that this bound is actually attained.

Proposition 33 (Pretzel knots). For $d \geq 0$ and odd integers $t_{0}, t_{1}, \ldots, t_{d}$ the corresponding pretzel link has determinant $\operatorname{det} P\left(t_{0}, t_{1}, \ldots, t_{d}\right)=i^{-d} S\left(t_{0}, t_{1}, \ldots, t_{d}\right)$, where $S$ is the elementary symmetric polynomial of degree $d$ in $d+1$ variables.

For any integer $d \geq 0$ we have $S\left(t_{0}, t_{1}, \ldots, t_{d}\right)=\sum_{k=0}^{k=d} \prod_{j \neq k} t_{j}$; for example, $S\left(t_{0}\right)=1$ and $S\left(t_{0}, t_{1}\right)=t_{0}+t_{1}$ and $S\left(t_{0}, t_{1}, t_{2}\right)=t_{0} t_{1}+t_{0} t_{2}+t_{1} t_{2}$. Fixing an even integer $d \geq 2$ and the parameter $t_{0}$, we obtain a $d$-dimensional twist lattice

$$
K: \mathbb{Z}^{d} \rightarrow \mathcal{K} \text { given by } K\left(z_{1}, \ldots, z_{d}\right)=P\left(t_{0}, 2 z_{1}-1, \ldots, 2 z_{d}-1\right),
$$

on which the squared determinant is a polynomial of degree $2 d$. Since its degree is unbounded, $\operatorname{det}^{2}$ cannot be a Vassiliev invariant.

Proof. The formula $\operatorname{det} P\left(t_{0}, t_{1}, \ldots, t_{d}\right)=i^{-d} S\left(t_{0}, t_{1}, \ldots, t_{d}\right)$ can be derived from the skein relation as follows. We set $N=\sum_{j}\left|t_{j}+1\right|$. If $N=0$, then $t_{j}=-1$ for all $j$, and the pretzel link $P\left(t_{0}, t_{1}, \ldots, t_{d}\right)$ coincides with the torus link $T(2, d+1)$. Its determinant is $i^{d}(d+1)$, which equals $i^{-d} S\left(t_{0}, t_{1} \ldots, t_{d}\right)$. If $N>0$, then there is a parameter $t_{j} \neq-1$, and the skein relation for $\operatorname{det} P$ yields

$$
\begin{aligned}
& \operatorname{det} P\left(t_{0}, \ldots, t_{j}, \ldots, t_{d}\right)-\operatorname{det} P\left(t_{0}, \ldots, t_{j} \pm 2, \ldots, t_{d}\right) \\
& \quad= \pm 2 i \cdot \operatorname{det} P\left(t_{0}, \ldots, \widehat{t_{j}}, \ldots, t_{d}\right) .
\end{aligned}
$$

This corresponds to the recursive property of the function $S$ :

$$
\begin{aligned}
& i^{-d} S\left(t_{0}, \ldots, t_{j} \ldots, t_{d}\right)-i^{-d} S\left(t_{0}, \ldots, t_{j} \pm 2, \ldots, t_{d}\right) \\
& \quad= \pm 2 i \cdot i^{-d+1} S\left(t_{0}, \ldots, \widehat{t_{j}}, \ldots, t_{d}\right) .
\end{aligned}
$$

The desired equality now follows by induction on $N$.

5.3. The determinant on torus knots. There is an alternative proof to show that $\operatorname{det}^{2}$ is not of finite type. It uses geometric sequences of torus knots as explained in Example 22. We include it in order to illustrate the characterization via geometric sequences given in Theorem 30 . 
Proposition 34 (Torus knots). Let $p$ and $q$ be coprime integers and let $K(z)=$ $T(p, q+z p)$ be the corresponding geometric sequence of torus knots.

(1) If $p$ is even, then $\operatorname{det}^{2} K(z)=(q+z p)^{2}$ is a quadratic polynomial in $z$.

(2) If $p$ is odd, then $\operatorname{det}^{2} K(z)$ is of period 2, taking alternating values 1 and $p^{2}$. In particular, $\operatorname{det}^{2}$ is not a Vassiliev invariant.

Proof. Up to factors $\pm t^{k}$, the Alexander polynomial of torus knots is given by

$$
\Delta T(p, q) \doteq \frac{\left(t^{p q}-1\right)(t-1)}{\left(t^{p}-1\right)\left(t^{q}-1\right)}
$$

(see [15, Chapter 11). Expanding this formula and evaluating it at $t=-1$ yields

$$
|\operatorname{det} T(p, q)|= \begin{cases}1 & \text { if both } p \text { and } q \text { are odd, } \\ |p| & \text { if } p \text { is odd and } q \text { is even, } \\ |q| & \text { if } p \text { is even and } q \text { is odd. }\end{cases}
$$

For the geometric sequence $K(z)=T(p, q+z p)$, two cases occur: If $p$ is even, then $\operatorname{det}^{2} K(z)=(q+z p)^{2}$ is quadratic in $z$. If $p$ is odd, however, then $\operatorname{det}^{2} K(z)$ takes alternating values 1 and $p^{2}$, and thus cannot be a polynomial in $z$.

\section{Characterization of Vassiliev invariants via polynomial growth}

As we have seen in the preceding example of the determinant, being a polynomial of degree $\leq m$ on every twist sequence does not imply that $v$ is a Vassiliev invariant. This section shows that the missing condition is that $v$ be uniformly bounded to have polynomial growth.

This motivates the definition of the following intermediate class of invariants:

Definition 35. An invariant $v: \mathcal{K} \rightarrow A$ is called locally polynomial (of degree $\leq m$ ) if it is a polynomial (of degree $\leq m$ ) on every twist sequence.

The condition that $v: \mathcal{K} \rightarrow A$ be locally polynomial of degree $\leq m$ can be restated as saying that $v$ vanishes on every singular knot with a chain of $m+1$ consecutive singularities, as shown in Figure 4.

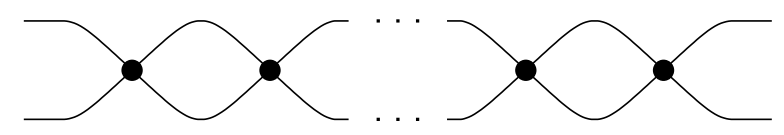

Figure 4. A chain of consecutive singularities

Suppose that $v$ is locally polynomial of degree $\leq m$. Then on every twist lattice $\mathbb{Z}^{d} \rightarrow \mathcal{K}$ the invariant $v$ is a polynomial of degree at most $d m$, but there may not be any global bound (independent of $d$ ). In order to discuss the idea of polynomial growth, we restrict our attention to rational invariants of knots in the sphere $\mathbb{S}^{3}$. Let $|K|$ be the crossing number of the knot $K$, i.e. the minimal number of crossings needed to represent $K$ by a planar diagram.

Definition 36. We say that an invariant $v: \mathcal{K} \mathbb{S}^{3} \rightarrow \mathbb{Q}$ has polynomial growth of degree $\leq m$ if it satisfies the inequality $|v(K)| \leq a|K|^{m}$ for all knots $K$ with sufficiently large crossing number. This is the same as saying that $v$ satisfies $|v(K)| \leq a|K|^{m}+b$ for all knots $K$ and some constants $a, b \in \mathbb{Q}$. 
Theorem 37. An invariant $v: \mathcal{K} \mathbb{S}^{3} \rightarrow \mathbb{Q}$ is a Vassiliev invariant of degree $\leq m$ if and only if $v$ is locally polynomial and has polynomial growth of degree $\leq m$.

Proof. To prove " $\Rightarrow$ ", assume that $v$ is a Vassiliev invariant of degree $\leq m$. By Theorem 28, $v$ is a polynomial of degree $\leq m$ on every twist sequence. The inequality $|v(K)| \leq a|K|^{m}+b$ was proven by D. Bar-Natan [3].

To prove " $\Leftarrow$ ", consider a twist lattice $\Phi: \mathbb{Z}^{d} \rightarrow \mathcal{K}$. It can be realized as a twist lattice of diagrams $D(z)$ having crossing numbers $c_{0}+2|z|$. Therefore on every twist lattice $\Phi$ the crossing number is bounded by $|\Phi(z)| \leq c_{0}+2|z|$ with some constant $c_{0} \in \mathbb{N}$.

We assume that $v$ is a polynomial on every twist sequence $\varphi: \mathbb{Z} \rightarrow \mathcal{K}$ and bounded by $|v(K)| \leq a|K|^{m}+b$. This implies that $v \circ \varphi: \mathbb{Z} \rightarrow \mathbb{Q}$ is a polynomial of degree $\leq m$. Given a twist lattice $\Phi: \mathbb{Z}^{d} \rightarrow \mathcal{K}$, the composition $v \circ \Phi: \mathbb{Z}^{d} \rightarrow \mathbb{Q}$ is hence a polynomial of degree $\leq d m$. Again by the boundedness argument, the degree of $v \circ \Phi$ must be $\leq m$. By Theorem 28 we conclude that $v$ is a Vassiliev invariant of degree $\leq m$.

Example 38. The crossing number has, of course, linear growth. The same holds for many other classical knot invariants, such as the unknotting number, the genus, the braid index, and the bridge number [10. They are not Vassiliev invariants, because they are not locally polynomial $[\underline{6}, 20$.

Example 39. The squared determinant is locally polynomial but has exponential growth: with respect to connected sums the determinant is multiplicative, which implies that $\operatorname{det}\left(K^{\sharp n}\right)$ grows exponentially while $\left|K^{\sharp n}\right|$ grows only linearly. This proves anew that the squared determinant is not a Vassiliev invariant.

Remark 40. A generalization from $\mathbb{S}^{3}$ to an arbitrary 3-manifold is possible. For example, the polynomial bound in the crossing number can be replaced by the condition that $v$ be dominated by $\max \left\{\left|v_{1}\right|, \ldots,\left|v_{k}\right|\right\}$, where $v_{1}, \ldots, v_{k}: \mathcal{K} M \rightarrow \mathbb{Q}$ are already known to be Vassiliev invariants of degree $\leq m$.

\section{VASSILIEV INVARIANTS WITH VALUES IN A TORSION GROUP}

The technique of geometric sequences and lattices can equally well be applied to invariants with values in an arbitrary abelian group $A$. The image of a polynomial function $f: \mathbb{Z}^{d} \rightarrow A$ is contained in a finitely generated subgroup of $A$. Without loss we can assume that $A$ itself is finitely generated; it thus splits into a free part and a torsion part. In this section we deal with the torsion case.

To analyze polynomial functions into a torsion group it suffices to study the case $A=\mathbb{Z} / p^{e}$, where $p$ is a prime and $e \geq 1$. The case $e=1$ is particularly simple:

Lemma 41. A map $f: \mathbb{Z} \rightarrow \mathbb{Z} / p$ is a polynomial of degree $<p^{\ell}$ if and only if it is of period $p^{\ell}$.

Proof. For $q=p^{\ell}$ we have

$$
\left(\partial^{q} f\right)(z)=\sum_{k=0}^{q}(-1)^{q-k}\left(\begin{array}{l}
q \\
k
\end{array}\right) \cdot f(z+k)=f(z+q)-f(z),
$$

because $0<k<q$ implies $\left(\begin{array}{l}q \\ k\end{array}\right) \equiv 0 \bmod p$. 
The situation is similar for maps $\mathbb{Z} \rightarrow \mathbb{Z} / p^{e}$ with $e \geq 2$, but unfortunately the relation between period and degree is less simple. We content ourselves with the following weaker characterization:

Lemma 42. A map $f: \mathbb{Z} \rightarrow \mathbb{Z} / p^{e}$ is a polynomial if and only if it is of period $p^{\ell}$ for some $\ell \geq 0$.

Proof. First observe that every polynomial function $\mathbb{Z} \rightarrow \mathbb{Z} / p^{e}$ is periodic. In view of Proposition 13 it suffices to show this for $\left(\begin{array}{l}z \\ k\end{array}\right) \bmod p^{e}$. For $q \in \mathbb{Z}$ we have $\left(\begin{array}{c}z+q \\ k\end{array}\right)-\left(\begin{array}{c}z \\ k\end{array}\right)=\frac{q}{k !} \cdot r$ with some remaining term $r \in \mathbb{Z}$. For $q=p^{\ell}$ with sufficiently large exponent $\ell$ we arrive at $\left(\begin{array}{c}z+q \\ k\end{array}\right) \equiv\left(\begin{array}{l}z \\ k\end{array}\right) \bmod p^{e}$ for all $z$.

Conversely, suppose that $f$ has period $q=p^{\ell}$ and values in $A \subset \mathbb{Z} / p^{e}$. The proof of the preceding lemma shows that $\partial^{q} f$ has values in $p A$. By iteration we see that $\partial^{q e} f=0$, which means that $f$ is a polynomial of degree $<q e$.

The condition can obviously be generalized to a $d$-dimensional lattice: a map $f: \mathbb{Z}^{d} \rightarrow \mathbb{Z} / p^{e}$ is a polynomial if and only if it is of period $p^{\ell}$ for some $\ell \geq 0$, i.e. $f(z)=f\left(z+p^{\ell} w\right)$ for all $z, w \in \mathbb{Z}^{d}$. Returning to the general case of a finite abelian group, we conclude the following criterion:

Corollary 43. Every polynomial $f: \mathbb{Z}^{d} \rightarrow A$ into a finite abelian group $A$ is periodic, i.e. there exists a period $q \geq 1$ such that $f(z)=f(z+q w)$ for all $z, w \in \mathbb{Z}^{d}$. If $q$ is the smallest such period, then the prime factors of $q$ divide $|A|$.

Example 44. We consider again the squared determinant, but this time modulo some integer $q$. Let $q=p 2^{m}$ with $p \geq 1$ odd. Since $\mathbb{Z} / q \cong \mathbb{Z} / p \times \mathbb{Z} / 2^{m}$, we have to consider two separate cases:

- Modulo any odd integer $p \geq 3$, the squared determinant $\operatorname{det}^{2}: \mathcal{K} \rightarrow \mathbb{Z} / p$ is not a Vassiliev invariant, because it is of period 2 on some geometric sequences, for example on torus knots $T(p, 1+z p)$ as in Proposition 34

- Modulo $2^{m}$, however, the determinant det $: \mathcal{K} \rightarrow \mathbb{Z} / 2^{m}$ is a Vassiliev invariant of degree $<m$, as can easily be seen from the Conway polynomial and the definition $\operatorname{det}(K)=\left.\nabla(K)\right|_{z=2 i}$.

The preceding example shows that the geometric sequence criterion can be useful in the torsion case as well. I do not know, however, whether this condition is also sufficient. If the group $A$ is torsion-free, then Lemma 29 states that polynomials $f: \mathbb{Z}^{d} \rightarrow A$ of degree $\leq m$ can be characterized by their behaviour on straight lines, from which we deduced Theorem 30 . This argument is not valid for torsion groups:

Example 45 (Straight lines). Let $p$ be a prime, and let $f: \mathbb{Z}^{d} \rightarrow \mathbb{Z} / p$ be given by $f(z)=z_{1} z_{2} \cdots z_{d} \bmod p$, which is a polynomial of degree $d$. On every straight line it is $p$-periodic and hence of degree $<p$. Since $d$ and $p$ are independent, the degree of $f$ cannot be determined by its behaviour on straight lines.

\section{TORSION IN BRAID GROUPS}

R.H. Fox and L.P. Neuwirth [14] defined the braid group $B_{n} S$ over an arbitrary surface $S$. As for Artin's braid group, the permutation of strands defines an epimorphism $\pi: B_{n} S \rightarrow \Sigma_{n}$ onto the symmetric group on $n$ points. The kernel $P_{n} S=\operatorname{ker}(\pi)$ is called the group of pure braids over the surface $S$.

We will use the technique of geometric sequences to prove that the torsion in the braid group over the sphere induces torsion at the level of Vassiliev invariants. 
8.1. Braid groups over the plane. Artin's braid group, which we have been using so far, is the braid group $B_{n} \mathbb{R}^{2}$ over the plane. It has the following wellknown presentation [1, 12, 4]:

$$
B_{n} \mathbb{R}^{2}=\left\langle\sigma_{1}, \ldots, \sigma_{n-1} \mid \begin{array}{cc}
\sigma_{i} \sigma_{j}=\sigma_{j} \sigma_{i} & \text { if }|i-j| \geq 2 \\
\sigma_{i} \sigma_{j} \sigma_{i}=\sigma_{j} \sigma_{i} \sigma_{j} & \text { if }|i-j|=1
\end{array}\right\rangle .
$$

There are several different proofs that $B_{n} \mathbb{R}^{2}$ is torsion-free:

- The configuration space of $n$ points in the plane is a $2 n$-dimensional manifold with fundamental group $B_{n} \mathbb{R}^{2}$. E. Fadell and L.P. Neuwirth [13 proved that it is an Eilenberg-MacLane space. This implies, by a standard argument of group cohomology, that $B_{n} \mathbb{R}^{2}$ is torsion-free. Their proof applies, in fact, to every surface that is different from the sphere $\mathbb{S}^{2}$ and the projective plane $\mathbb{P}^{2}$.

- Using Seifert fibre spaces, K. Murasugi 17] proved that $B_{n} \mathbb{R}^{2}$ is torsion-free and classified the torsion elements in $B_{n} \mathbb{S}^{2}$ and $B_{n} \mathbb{P}^{2}$ (see below).

- J.L. Dyer 8 gave an algebraic proof based on Artin's faithful representation $B_{n} \mathbb{R}^{2} \rightarrow \operatorname{Aut}\left(F_{n}\right)$ of braids as automorphisms of a free group.

- P. Dehornoy [7] constructed a left-invariant linear ordering on $B_{n} \mathbb{R}^{2}$, which implies, among other things, that $B_{n}$ is torsion-free.

8.2. Braid groups over the sphere. For the braid group over the sphere, E. Fadell and J.van Buskirk [12] obtained the following presentation:

$$
B_{n} \mathbb{S}^{2}=\left\langle\sigma_{1}, \ldots, \sigma_{n-1} \mid \begin{array}{cc}
\sigma_{i} \sigma_{j}=\sigma_{j} \sigma_{i} & \text { if }|i-j| \geq 2 \\
\sigma_{i} \sigma_{j} \sigma_{i}=\sigma_{j} \sigma_{i} \sigma_{j} & \text { if }|i-j|=1 \\
\sigma_{1} \sigma_{2} \cdots \sigma_{n-1} \sigma_{n-1} \cdots \sigma_{2} \sigma_{1}=1
\end{array}\right\rangle .
$$

The additional relation $r_{n}=\sigma_{1} \sigma_{2} \cdots \sigma_{n-1}^{2} \cdots \sigma_{2} \sigma_{1}$ is depicted in Figure 5 a below. Unlike Artin's braid group $B_{n} \mathbb{R}^{2}$, the braid group $B_{n} \mathbb{S}^{2}$ over the sphere has torsion elements. For example we have $B_{2} \mathbb{S}^{2} \cong \mathbb{Z} / 2$ and $B_{3} \mathbb{S}^{2} \cong \mathbb{Z} / 3 \rtimes \mathbb{Z} / 4$. K. Murasugi [17] proved that $B_{n} \mathbb{S}^{2}$ is infinite for $n \geq 4$ and classified all its torsion elements up to conjugation (see below).

In view of geometric sequences we are mainly interested in the pure braid group $P_{n} \mathbb{S}^{2}$. Here the obvious torsion candidate is the pure braid $\tau_{n}$, corresponding to a full twist of all $n$ strands as depicted in Figure 5 b.

(a)

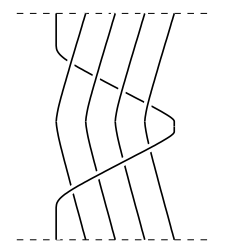

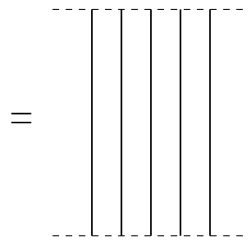

(b)

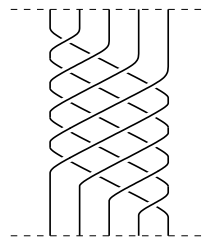

FiguRe 5. (a) Braid relation over the sphere, (b) braid of order 2

The braid $\tau_{n}$ is easily seen to satisfy $\tau_{n}^{2}=1$. Using the Dirac twist explained in 9.1. this follows from the fact that $\pi_{1} \mathrm{SO}(3) \cong \mathbb{Z} / 2$. In fact, this observation was used by P.A.M. Dirac in the 1930's to visualize spin phenomena. The exact formulation in terms of braid groups was first investigated by M.H.A. Newman [18].

Theorem 46. For $n \geq 3$, the pure braid group $P_{n} \mathbb{S}^{2}$ contains a unique torsion element, namely the braid $\tau_{n}=\left(\sigma_{1} \sigma_{2} \cdots \sigma_{n-1}\right)^{n}$ of order 2 . 
Proof. To show that $\tau_{n} \neq 1$, we consider the abelianization $v_{n}: B_{n} \mathbb{S}^{2} \rightarrow \mathbb{Z} / 2 n-2$ given by the sum of exponents modulo $2 n-2$. The equality $v_{n}\left(\tau_{n}\right)=n(n-1)$ proves that $\tau_{n} \neq 1_{n}$ for all $n \geq 3$ odd. For $n \geq 4$ even, we consider the group homomorphism $P_{n} \mathbb{S}^{2} \rightarrow P_{n-1} \mathbb{S}^{2}$ given by deleting the last strand. Since $\tau_{n}$ is mapped to $\tau_{n-1}$, this shows that $\tau_{n} \neq 1_{n}$ for all $n \geq 3$.

The braid group $B_{n} \mathbb{S}^{2}$ contains the following obvious torsion elements:

$$
\begin{array}{ll}
\alpha_{n}=\sigma_{1} \sigma_{2} \cdots \sigma_{n-1} & \text { has order } 2 n, \\
\beta_{n}=\sigma_{1} \sigma_{2} \cdots \sigma_{n-1} \sigma_{1} & \text { has order } 2(n-1), \\
\gamma_{n}=\sigma_{1} \sigma_{2} \cdots \sigma_{n-2} \sigma_{1} & \text { has order } 2(n-2) .
\end{array}
$$

To see this, we consider the natural epimorphism $\pi: B_{n} \mathbb{S}^{2} \rightarrow \Sigma_{n}$ :

$$
\begin{array}{lll}
\pi\left(\alpha_{n}\right)=(123 \ldots n) & \text { has order } n \quad \text { and } \alpha_{n}^{n}=\tau_{n} \quad \text { has order 2, } \\
\pi\left(\beta_{n}\right)=(134 \ldots n) & \text { has order } n-1 & \text { and } \beta_{n}^{n-1}=\tau_{n} \text { has order } 2, \\
\pi\left(\gamma_{n}\right)=(134 \ldots n-1) & \text { has order } n-2 & \text { and } \gamma_{n}^{n-2}=\tau_{n} \text { has order } 2 .
\end{array}
$$

K. Murasugi [17] proved that each torsion element in $B_{n} \mathbb{S}^{2}$ is conjugate to $\alpha_{n}^{k}$ or $\beta_{n}^{k}$ or $\gamma_{n}^{k}$ for a suitable exponent $k$. Hence every torsion element in $P_{n} \mathbb{S}^{2}$ must be conjugate to $\tau_{n}$. Since $\tau_{n}$ is central in $B_{n} \mathbb{S}^{2}$, we conclude that $\tau_{n}$ is the unique torsion element in $P_{n} \mathbb{S}^{2}$.

Lemma 47. Let $A$ be an abelian group without 2-torsion and $v: B_{n} \mathbb{S}^{2} \rightarrow A$ an invariant of finite type. Then $v$ cannot distinguish the braids $\tau_{n}$ and $1_{n}$.

Proof. Since $\tau_{n}$ is a pure braid of order 2, the sequence $\tau_{n}^{z}$ is geometric of period 2 , and $v\left(\tau_{n}^{z}\right)$ is a polynomial in $z$ of period 2. We may assume that $A$ is finitely generated. Since $A$ splits into a free part and a torsion part, it suffices to consider two cases: If $A$ is torsion free, then $v\left(\tau_{n}^{z}\right)$ is a bounded polynomial and hence constant. If $A$ is finite of odd order, then the same conclusion can be drawn from Corollary 43

Note that the abelianization $v_{n}: B_{n} \mathbb{S}^{2} \rightarrow \mathbb{Z} / 2 n-2$ is a Vassiliev invariant of degree 1 , and the distinction between $\tau_{n}$ and $1_{n}$ is due to the 2 -torsion part. The next section is devoted to generalizing this construction from braids to knots.

\section{Applichtion to knots in $\mathbb{S}^{1} \times \mathbb{S}^{2}$}

In this section we show that there exist knots in $\mathbb{S}^{1} \times \mathbb{S}^{2}$ that cannot be distinguished by any rational Vassiliev invariant. In order to construct such examples we use the Dirac twist to define an involution $\theta$ on the set of knots.

9.1. The Dirac twist. For $t \in[0,1]$ let $\rho_{t}: \mathbb{R}^{3} \rightarrow \mathbb{R}^{3}$ be a rotation of $2 \pi t$ around some chosen axis. The loop $\rho:[0,1] \rightarrow \mathrm{SO}(3)$ given by $t \mapsto \rho_{t}$ represents the non-trivial element in $\pi_{1} \mathrm{SO}(3) \cong \mathbb{Z} / 2$. Define $\theta:[0,1] \times \mathbb{S}^{2} \rightarrow[0,1] \times \mathbb{S}^{2}$ by $\theta(t, s)=\left(t, \rho_{t}(s)\right)$. The homeomorphism $\theta$ fixes the boundary $\{0,1\} \times \mathbb{S}^{2}$ pointwise, and its square $\theta^{2}$ is isotopic to the identity relative to the boundary. We call the homeomorphism $\theta$ the Dirac twist of $[0,1] \times \mathbb{S}^{2}$.

Remark 48. The Dirac twist is the 3-dimensional analogue of the 2-dimensional Dehn twist $[0,1] \times \mathbb{S}^{1} \rightarrow[0,1] \times \mathbb{S}^{1}$ given by $(t, s) \mapsto\left(t, \rho_{t}(s)\right)$ where $\rho_{t}$ is the rotation of the plane by $2 \pi t$. There are higher-dimensional analogues as well. The remarkable difference is that $\pi_{1} \mathrm{SO}(2) \cong \mathbb{Z}$, whereas $\pi_{1} \mathrm{SO}(n)$ is of order 2 for $n \geq 3$. 
Remark 49. For every braid $\sigma \in B_{n} \mathbb{S}^{2}$ we have $\theta(\sigma)=\sigma \tau_{n}$. This relationship is interesting in two ways: The isotopy from $\theta^{2}$ to the identity transforms $\tau_{n}^{2}$ into the trivial braid $1_{n}$, which is a nice geometric proof that $\tau_{n}$ has order at most 2 . On the other hand $\tau_{n} \neq 1_{n}$ witnesses that $\theta$ is not isotopic to the identity.

9.2. Twin knots. In order to apply the Dirac twist to knots in a 3-manifold $M$ we consider an embedding $S:[0,1] \times \mathbb{S}^{2} \hookrightarrow M$, i.e. a thickened sphere in $M$. We define $\theta_{S}: M \rightarrow M$ by $\theta_{S}(x)=S \theta S^{-1}(x)$ if $x$ is in the image of $S$, and $\theta_{S}(x)=x$ otherwise. Clearly, $\theta_{S}$ is a homeomorphism, and $\theta_{S}^{2}$ is isotopic to the identity of $M$. Moreover, the isotopy class of $\theta_{S}$ depends only on the isotopy class of $S$.

Definition 50. Given a thickened sphere $S$ in a manifold $M$, we call $\theta_{S}: M \rightarrow M$ the Dirac twist associated with $S$. For a knot $K$ in $M$ we call $\theta_{S} K$ its twin knot. We will simply write $\theta: M \rightarrow M$ when the embedding $S$ is understood.

Lemma 51. If $A$ is an abelian group without 2-torsion, then Vassiliev invariants $\mathcal{K} M \rightarrow A$ cannot distinguish a knot $K$ from its twin $\theta K$.

Proof. After an isotopy we can assume that the thickened sphere $S$ intersects $K$ in a trivial braid on $n$ strands. Thus the application of $\theta^{z}$ corresponds to inserting the braid $\tau_{n}^{z}$, which shows that $\theta^{z} K$ is a geometric sequence. Furthermore, $\theta^{2} K=K$, because $\theta^{2}$ is isotopic to the identity and we regard knots only up to isotopy.

Suppose that $v: \mathcal{K} M \rightarrow A$ is a Vassiliev invariant. By construction, $v\left(\theta^{z} K\right)$ is a polynomial in $z$ and of period 2. As we have already seen in the proof of Lemma 47 $v\left(\theta^{z} K\right)$ must be constant if $A$ has no 2-torsion. Hence $v(K)=v(\theta K)$.

Remark 52. The preceding lemma does not say that $K$ and $\theta K$ are distinct. For example, if the sphere $S$ bounds a ball in $M$, then $\theta$ is isotopic to the identity of $M$, and of course $\theta K=K$. If the sphere $S$ is essential, however, chances are that $\theta K \neq K$. Alas, the distinction between $K$ and $\theta K$ is quite subtle: by construction the spaces $(M, K)$ and $(M, \theta K)$ are homeomorphic. Moreover, $K$ and $\theta K$ are homotopic in $M$. This means that the classical invariants of algebraic topology do not distinguish $K$ and $\theta K$.

9.3. Twin knots in $\mathbb{S}^{1} \times \mathbb{S}^{2}$. It remains to be shown that $K$ and $\theta K$ are actually distinct in some cases. The simplest non-trivial example is the manifold $\mathbb{S}^{1} \times \mathbb{S}^{2}$.

Theorem 53. Let $u: H_{1}\left(\mathbb{S}^{1} \times \mathbb{S}^{2}\right) \rightarrow \mathbb{Z}$ be an isomorphism. For every knot $K$ in $\mathbb{S}^{1} \times \mathbb{S}^{2}$ having homology class $u(K) \in\{ \pm 3, \pm 5, \pm 7, \ldots\}$, the following hold:

(1) The knot $K$ and its twin $\theta K$ are distinguished by a suitable Vassiliev invariant $\mathcal{K}\left(\mathbb{S}^{1} \times \mathbb{S}^{2}\right) \rightarrow \mathbb{Z} / 2$ of degree 1 .

(2) The knot $K$ and its twin $\theta K$ cannot be distinguished by any Vassiliev invariant $\mathcal{K}\left(\mathbb{S}^{1} \times \mathbb{S}^{2}\right) \rightarrow A$ if the abelian group $A$ has no 2 -torsion.

In particular, rational invariants of finite type do not distinguish all knots in $\mathbb{S}^{1} \times \mathbb{S}^{2}$.

Assertion (2) holds for all twin knots and was proven in Lemma 51 above. The subtle point is to distinguish $K$ and $\theta K$. A proof modelled on the abelianization $v_{n}: B_{n} \mathbb{S}^{2} \rightarrow \mathbb{Z} / 2 n-2$ has been sketched in [11. A complete proof will be given in the next section, where we construct a universal Vassiliev invariant of degree 1 by combinatorial integration.

Remark 54. It seems plausible that $K \neq \theta K$ also holds for knots having homology class $u(K) \in\{ \pm 4, \pm 6, \pm 8, \ldots\}$, as well as for certain knots with homology class 
$0, \pm 1, \pm 2$. Suitable invariants can probably be found among Vassiliev invariants $\mathcal{K}\left(\mathbb{S}^{1} \times \mathbb{S}^{2}\right) \rightarrow \mathbb{Z} / 2$ of degree $\geq 2$. A more systematic treatment of torsion invariants would certainly be desirable, but it seems difficult in general.

One can also try to generalize the construction to other reducible 3-manifolds:

Question 55. Suppose that $M=M_{1} \sharp M_{2}$ is a non-trivial connected sum and the thickened sphere $S$ bounds $M_{1}$ on one side and $M_{2}$ on the other. Under which condition is a knot $K$ distinct from its twin $\theta_{S} K$ ? How can we construct invariants that distinguish $K$ and $\theta_{S} K$ ?

\section{Combinatorial integration In $\mathbb{S}^{1} \times \mathbb{S}^{2}$}

In his thesis [16], J. Lieberum has constructed a Kontsevich isomorphism for knots in $\mathbb{S}^{1} \times \mathbb{S}^{2}$. Unfortunately, the Kontsevich integral and its generalizations only work in characteristic zero, and thus ignore the torsion part. In order to construct torsion invariants, we will hence carry out a combinatorial integration. In view of Theorem 53 and to keep the argument as simple as possible, we will restrict our attention to weight systems of degree 1.

10.1. Surgery presentation for knots in $\mathbb{S}^{1} \times \mathbb{S}^{2}$. We study knots in $M=\mathbb{S}^{1} \times \mathbb{S}^{2}$ via their surgery presentation. Let $\mathbb{S}^{2}=\mathbb{D}^{2} \cup P$, where $\mathbb{D}^{2}$ is the open disk and $P$ consists of a single point. This yields a decomposition of our manifold $M$ into a solid torus $T=\mathbb{S}^{1} \times \mathbb{D}^{2}$ and a circle $\mathbb{S}^{1} \times P$. The inclusion $T \subset M$ induces a surjection $\mathcal{K} T \rightarrow \mathcal{K} M$, which simply means that every knot in $M$ can be represented as a knot in $T$. Of course, this representation is not unique:

Definition 56. We say that two knots $K$ and $K^{\prime}$ in $T$ differ by a Kirby move if they coincide outside a sector $U=[a, b] \times \mathbb{D}^{2}$ of $T$, inside of which $K \cap U$ is a trivial braid on $k$ strands, whereas $K^{\prime} \cap U$ is given by the braid $r_{k}=\sigma_{1} \sigma_{2} \cdots \sigma_{k-1}^{2} \cdots \sigma_{2} \sigma_{1}$. The Kirby move $K \rightleftharpoons K^{\prime}$ thus corresponds to Figure 5 h.

Lemma 57. Two knots in the solid torus $T$ represent the same knot in $\mathbb{S}^{1} \times \mathbb{S}^{2}$ if and only if they are related by a sequence of isotopies and Kirby moves.

10.2. Chord diagrams of degree one. We will deal with Vassiliev invariants in the manifold $M=\mathbb{S}^{1} \times \mathbb{S}^{2}$ and in the solid torus $T=\mathbb{S}^{1} \times \mathbb{D}^{2}$. As before, let $\mathbb{Z} \mathcal{K}=\mathcal{F}_{0} \supset \mathcal{F}_{1} \supset \mathcal{F}_{2} \supset \cdots$ be the Vassiliev filtration of knots (in $M$ or in $T$ respectively). Our aim is to understand the quotients $\mathcal{F}_{n} / \mathcal{F}_{n+1}$ by means of chord diagrams. To this end we consider the natural maps of the following diagram:

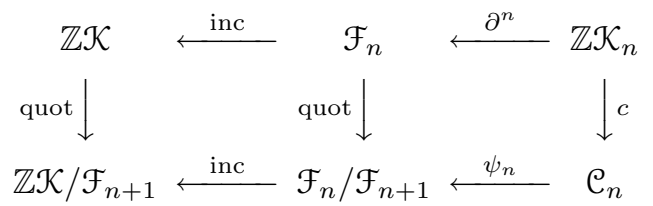

Here $\mathcal{C}_{n}$ is the module of chord diagrams, i.e. $n$-singular knots modulo homotopy, and the forgetful map $c: \mathbb{Z} \mathcal{K}_{n} \rightarrow \mathcal{C}_{n}$ sends each $n$-singular knot to its chord diagram. The map $\partial^{n}$ assigns to each $n$-singular knot its resolution as explained in \$1.2. Modulo $\mathcal{F}_{n+1}$ it passes to the quotient $\mathcal{C}_{n}$ and induces $\psi_{n}: \mathcal{C}_{n} \rightarrow \mathcal{F}_{n} / \mathcal{F}_{n+1}$.

In order to simplify matters we will only analyze the case $n=1$. For integers $a, b$ let $C(a, b)$ be the chord diagram depicted in Figure 6, It is obviously symmetric in 


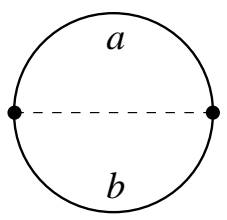

FIGURE 6 .

the sense that $C(a, b)=C(b, a)$. Let $\mathcal{C}_{1}$ be the $\mathbb{Z}$-module with basis $C(a, b)$ indexed by $a, b \in \mathbb{Z}, a \leq b$.

The map $c: \mathcal{K}_{1} \rightarrow \mathcal{C}_{1}$ associates to each 1-singular knot $K \bullet$ its chord diagram $c\left(K_{\bullet}\right)$ : cutting the singularity according to the model $\left.\chi \rightarrow\right)($ produces two oriented curves $K_{1}$ and $K_{2}$ with homology classes $u\left(K_{1}\right)=a$ and $u\left(K_{2}\right)=b$, and we obtain $c\left(K_{\bullet}\right)=C(a, b)$.

Proposition 58. For the solid torus $T=\mathbb{S}^{1} \times \mathbb{D}^{2}$, the map $\psi_{1}^{T}: \mathcal{C}_{1} \rightarrow \mathcal{F}_{1} T / \mathcal{F}_{2} T$ is surjective and its kernel contains the following obvious relation:

(1) Framing invariance: $\quad C(a, b)=0 \quad$ whenever $a=0$ or $b=0$.

For $M=\mathbb{S}^{1} \times \mathbb{S}^{2}$, the map $\psi_{1}^{M}: \mathcal{C}_{1} \rightarrow \mathcal{F}_{1} M / \mathcal{F}_{2} M$ is again surjective. Its kernel contains relation (1) and the following additional relation:

(2) Kirby invariance: $\quad \sum_{0<k<n} C(k, n-k)=0 \quad$ for $n \geq 0$, and analogously $\quad \sum_{n<k<0} C(k, n-k)=0 \quad$ for $n \leq 0$.

Proof. Surjectivity is clear. The first relation follows from Reidemeister moves of type 1 . The second relation follows from the Kirby move depicted in Figure 5 a.

10.3. Torsion at the level of chord diagrams. We will show that the relations of Proposition 58 generate the whole kernel, but first we have a closer look at the quotients. Let $\mathcal{A}_{1} T$ be the quotient of $\mathcal{C}_{1}$ by relation (1), and let $\mathcal{A}_{1} M$ be the quotient of $\mathcal{C}_{1}$ by relations (1) and (2). The maps $\psi_{1}^{T}$ resp. $\psi_{1}^{M}$ defined above thus induce $\Psi_{1}^{T}: \mathcal{A}_{1} T \rightarrow \mathcal{F}_{1} T / \mathcal{F}_{2} T$ resp. $\Psi_{1}^{M}: \mathcal{A}_{1} M \rightarrow \mathcal{F}_{1} M / \mathcal{F}_{2} M$, which will turn out to be isomorphisms. We begin by stating the following observation:

Proposition 59. The $\mathbb{Z}$-module $\mathcal{A}_{1} T$ is free with basis $C(a, b)$ indexed by all pairs $a, b \in \mathbb{Z} \backslash\{0\}$ with $a \leq b$.

Let $\bar{C}(a, b)$ be the image of $C(a, b)$ in $\mathcal{A}_{1} M$ and define

$$
\begin{array}{ll}
t_{n}=\sum_{0<k<n} k \cdot \bar{C}(k, n-k) & \text { for } n \geq 0 \text { and } \\
t_{n}=\sum_{n<k<0} k \cdot \bar{C}(k, n-k) & \text { for } n \leq 0 \text { respectively. }
\end{array}
$$

Obviously $t_{n}=0$ for $|n| \leq 1$. Moreover, relation (2) implies $t_{n}=0$ for $n$ even, and $2 t_{n}=0$ for $n$ odd. More precisely, we have

Proposition 60. The $\mathbb{Z}$-module $\mathcal{A}_{1} M$ splits into a free part and a torsion part. The free part has basis $\bar{C}(a, b)$ indexed by all pairs $a, b \in \mathbb{Z} \backslash\{0\}$ with $a \leq b-2$. The torsion part is a vector space over $\mathbb{Z} / 2$ with basis $t_{n}$ indexed by $n \in\{ \pm 3, \pm 5, \pm 7, \ldots\}$.

Proof. The module $\mathcal{A}_{1} T=\bigoplus_{n} \mathcal{A}_{1, n} T$ is graded with respect to the homology class $n=a+b$. Relation (2) respects this grading, so the quotient $\mathcal{A}_{1} M=\bigoplus_{n} \mathcal{A}_{1, n} M$ 
is also graded. Moreover, relation (2) is empty for $n=0$ and for $n= \pm 1$. In these cases $\mathcal{A}_{1, n} M \cong \mathcal{A}_{1, n} T$ has basis $\bar{C}(a, n-a)$ with $a \leq \frac{n-2}{2}$, as claimed.

For ease of notation we will assume $n \geq 2$ in the sequel, the case $n \leq-2$ being analogous. The module $\mathcal{A}_{1, n} T$ has basis $C(a, n-a)$ with $a \leq \frac{n}{2}$ and $a \neq 0$. Two cases occur, according to whether $n$ is even or odd:

- If $n=2 m$ is even, then we obtain a new basis for $\mathcal{A}_{1, n} T$ by replacing $C(m, m)$ with $s_{n}=\sum_{0<k<n} C(k, n-k)$. In this new basis, relation (2) becomes $s_{n}=0$. Thus the quotient $\mathcal{A}_{1, n} M$ has basis $\bar{C}(a, n-a)$ with $a \leq \frac{n-2}{2}$ and $a \neq 0$.

- If $n=2 m+1$ is odd, then we obtain a new basis for $\mathcal{A}_{1, n} T$ by replacing $C(m, m+1)$ with $s_{n}=\sum_{0<k \leq m} C(k, n-k)$. In this new basis, relation (2) becomes $2 s_{n}=0$. Moreover, $s_{n}$ maps to $t_{n}$ in the quotient $\mathcal{A}_{1} M$. This means that $\mathcal{A}_{1, n} M$ has one element $t_{n}$ of order 2 , while the free part has basis $\bar{C}(a, n-a)$ with $a \leq \frac{n-3}{2}$ and $a \neq 0$.

This completes the proof on the module structure of $\mathcal{A}_{1} M$.

10.4. Integrating invariants of degree one. Let $A$ be an abelian group. A Vassiliev invariant $v: \mathcal{K} \rightarrow A$ of degree 1 defines a weight system $w: \mathcal{A}_{1} \rightarrow A$ by setting $w=v \circ \Psi_{1}$. Conversely, we call $v$ an integral of $w$. It is natural to ask whether there exists an integral for every weight system $w: \mathcal{A}_{1} \rightarrow A$.

This can be reformulated as follows: a universal Vassiliev invariant of degree 1 is a map $Z_{1}: \mathcal{K} \rightarrow \mathcal{A}_{1}$ that vanishes on $\mathcal{F}_{2}$ and satisfies $Z_{1} \Psi_{1}=$ id. Given a weight system $w$, this allows us to define an integral $v=w \circ Z_{1}$. In particular, such a map $Z_{1}$ restricts to $\mathcal{F}_{1}$ and induces an isomorphism $\Phi_{1}: \mathcal{F}_{1} / \mathcal{F}_{2} \rightarrow \mathcal{A}_{1}$ that is inverse to $\Psi_{1}: \mathcal{A}_{1} \rightarrow \mathcal{F}_{1} / \mathcal{F}_{2}$.

Theorem 61. For the manifold $M=\mathbb{S}^{1} \times \mathbb{S}^{2}$ there exists a $\mathbb{Z}$-universal Vassiliev invariant $Z_{1}^{M}: \mathcal{K} M \rightarrow \mathcal{A}_{1} M$ of degree 1 .

To prove this theorem we present knots in $M$ by knots in the solid torus $T$ modulo Kirby moves. The following folklore lemma constructs a universal Vassiliev invariant of degree 1 for knots in the solid torus.

Lemma 62. For the solid torus $T=\mathbb{S}^{1} \times \mathbb{D}^{2}$ there exists a $\mathbb{Z}$-universal Vassiliev invariant $Z_{1}^{T}: \mathcal{K} T \rightarrow \mathcal{A}_{1} T$ of degree 1 .

Proof. Let $D$ be a knot diagram on the annulus. Cutting a crossing $p$ yields two oriented curves $D^{\prime}$ and $D^{\prime \prime}$ with homology classes $p^{\prime}=u\left(D^{\prime}\right)$ and $p^{\prime \prime}=u\left(D^{\prime \prime}\right)$. This allows us to define the sum $f(D)=\sum_{p} \varepsilon(p) C\left(p^{\prime}, p^{\prime \prime}\right)$ over all crossings $p$ of $D$. Here $\varepsilon(p)$ is the sign of the crossing, defined by $\varepsilon(\swarrow)=+1$ and $\varepsilon(\chi)=-1$.

The value $f(D)$ in $\mathcal{A}_{1} T$ is invariant under Reidemeister moves and thus defines a knot invariant $f: \mathcal{K} T \rightarrow \mathcal{A}_{1} T$. By construction we have $f(\swarrow)-f(\searrow)=2 c(\nearrow)$. Since $\mathcal{A}_{1} T$ is a free $\mathbb{Z}$-module, there exists a knot invariant $Z_{1}^{T}: \mathcal{K} T \rightarrow \mathcal{A}_{1} T$ satisfying $Z_{1}^{T}(\swarrow)-Z_{1}^{T}(\chi)=c(\swarrow)$. This shows that $Z_{1}^{T}$ is a Vassiliev invariant of degree 1 satisfying $Z_{1}^{T} \Psi_{1}^{T}=$ id.

Lemma 63. The composition $\bar{Z}_{1}^{T}: \mathcal{K} T \rightarrow \mathcal{A}_{1} T \rightarrow \mathcal{A}_{1} M$ is invariant under Kirby moves. Thus $\bar{Z}_{1}^{T}$ induces a $\mathbb{Z}$-universal Vassiliev invariant $Z_{1}^{M}: \mathcal{K} M \rightarrow \mathcal{A}_{1} M$ of degree 1 , for the manifold $M=\mathbb{S}^{1} \times \mathbb{S}^{2}$. 
Proof. Let $K$ and $K^{\prime}$ be two knots that are identical outside a sector $U \subset T$, inside of which they are given by the braids $1_{k}$ and $r_{k}$ as depicted in Figure 5 a. We have to show that $Z_{1}^{T}(K) \equiv Z_{1}^{T}\left(K^{\prime}\right)$ modulo relation (2) of Proposition 58

Let $n=u(K)$ be the homology class of $K$ and $K^{\prime}$. To simplify notation we will assume $n \geq 0$, the case $n \leq 0$ being analogous. Furthermore, we can assume that the leftmost strand in $U$ is oriented positively. If necessary, we can achieve this by the looping manœuvre of Figure 7. This does not alter the knots $K$ and $K^{\prime}$, but ensures that the Kirby move $K \rightleftharpoons K^{\prime}$ is performed on a positive strand.
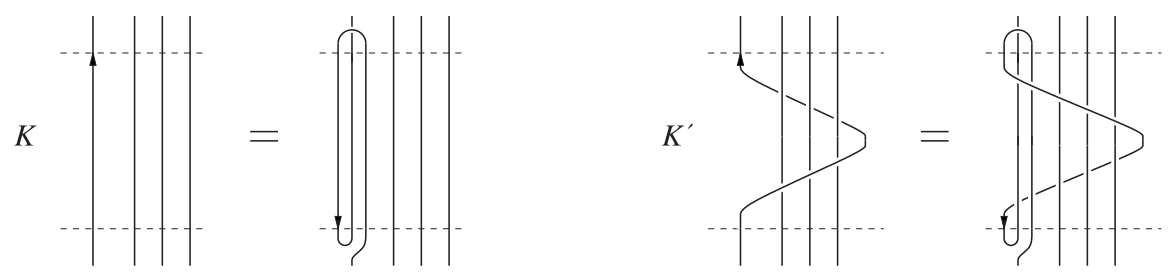

FIgURE 7. Transforming a negative Kirby move into a positive one

Beginning with the leftmost strand, we number the strands with $1, \ldots, k$ in the order they are visited when travelling along the knot. After a permutation of strands we can assume that they are visited in this order from left to right. Such a permutation alters the diagrams but not the knots $K$ and $K^{\prime}$.

Let $k_{\downarrow}$ resp. $k_{\uparrow}$ be the number of strands with positive resp. negative orientation. Thus $k=k_{\downarrow}+k_{\uparrow}$ and $n=k_{\downarrow}-k_{\uparrow}$. We prove $\bar{Z}_{1}^{T}(K)=\bar{Z}_{1}^{T}\left(K^{\prime}\right)$ by induction on the number $k_{\uparrow}$ of negative strands. The case $k_{\uparrow}=0$ is clear: all strands are oriented the same way and the difference $Z_{1}^{T}(K)-Z_{1}^{T}\left(K^{\prime}\right)$ is given by $\sum_{0<i<n} \bar{C}(i, n-i)$. Kirby invariance is thus guaranteed by relation (2) of Proposition 58 .

In the general case we will simplify $K$ and $K^{\prime}$ by simultaneously changing crossings outside the sector $U$. This alters the knots $K$ and $K^{\prime}$ but not the difference $Z_{1}^{T}(K)-Z_{1}^{T}\left(K^{\prime}\right)$, because $Z_{1}^{T}$ is a Vassiliev invariant of degree 1 .

In the case $k_{\uparrow} \geq 1$ there exists an index $i$ such that the strands $i$ and $i+1$ have opposite orientations. Let $i$ be maximal with this property. Then some crossing changes and Reidemeister moves outside $U$ lead to a situation as in Figure 8 The indicated Reidemeister moves then reduce the number $k_{\uparrow}$ by one. (This also holds in the exceptional case $i=1$, where we necessarily have $k=2$ and $n=0$. After some crossing changes outside $U$ we can reduce this to $k=0$.)
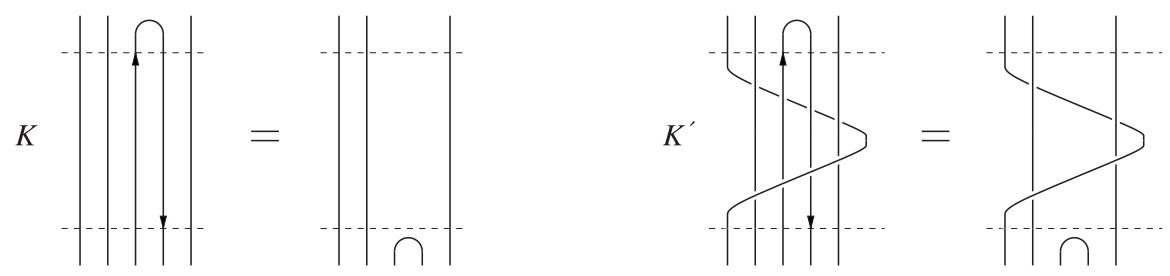

FIGURE 8. Reducing the number $k_{\uparrow}$ of negative strands

By induction on $k_{\uparrow}$ we conclude that $Z_{1}^{T}(K) \equiv Z_{1}^{T}\left(K^{\prime}\right)$ modulo relation (2), which means that $\bar{Z}_{1}^{T}$ is invariant under Kirby moves. Hence $\bar{Z}_{1}^{T}$ induces a universal Vassiliev invariant $Z_{1}^{M}: \mathcal{K} M \rightarrow \mathcal{A}_{1} M$ for our manifold $M=\mathbb{S}^{1} \times \mathbb{S}^{2}$. 
10.5. Distinguishing twin knots. In order to distinguish twin knots in $\mathbb{S}^{1} \times \mathbb{S}^{2}$ we study the behaviour of the universal Vassiliev invariant $Z_{1}^{M}$ under Dirac twists:

Lemma 64. Suppose that $K$ is a knot in $M=\mathbb{S}^{1} \times \mathbb{S}^{2}$ having homology class $n$. Then $Z_{1}^{M}(K)-Z_{1}^{M}(\theta K)=t_{n}$, with $t_{n} \in \mathcal{A}_{1} M$ as defined in $\$ 10.3$.

Proof. Define $t: \mathcal{K}\left(\mathbb{S}^{1} \times \mathbb{S}^{2}\right) \rightarrow \mathcal{A}_{1} M$ by $t(K)=Z_{1}^{M}(K)-Z_{1}^{M}(\theta K)$. Since $Z_{1}^{M}$ is of degree 1 , the difference $t$ is of degree 0 , hence invariant under crossing changes.

It thus suffices to prove $t(K)=t_{n}$ for any one knot $K$ with homology class $n$. In the case $|n| \leq 1$ we clearly have $t(K)=0$, which coincides with $t_{n}=0$. For the rest of this proof we will assume $n \geq 2$, the case $n \leq-2$ being analogous.

Let $K$ be a closed $n$-braid in the torus. Application of $\theta$ inserts the braid $\tau_{n}$ as depicted in Figure 9. We number the strands with $1, \ldots, n$ in the order that they are visited when travelling along the knot. The difference $Z_{1}^{M}(K)-Z_{1}^{M}(\theta K)$ is calculated by changing the marked crossings, one for each pair $(i, j)$ with $1 \leq$ $i<j \leq n$. Since all strands are oriented the same way, this gives a contribution $\bar{C}(j-i, n-j+i)$. Summing over all pairs yields $t_{n}$.

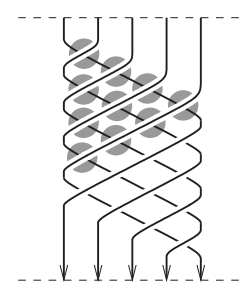

FIGURE 9.

Proof of Theorem 53. Suppose that $K$ is a knot in $\mathbb{S}^{1} \times \mathbb{S}^{2}$ having an odd homology class $n=u(K)$ with $|n| \geq 3$. It remains to be shown that $K$ can be distinguished from its twin knot $\theta K$ by a Vassiliev invariant $v: \mathcal{K}\left(\mathbb{S}^{1} \times \mathbb{S}^{2}\right) \rightarrow \mathbb{Z} / 2$ of degree 1 .

We will assume $n \geq 3$, the case $n \leq-3$ being analogous. By the preceding lemma we have $Z_{1}^{M}(K)-Z_{1}^{M}(\theta K)=t_{n}$. We define a weight system $w: \mathcal{A}_{1} M \rightarrow \mathbb{Z} / 2$ by $w(\bar{C}(a, b))=1$ if $\{a, b\}=\{1, n-1\}$, and $w(\bar{C}(a, b))=0$ otherwise. Since $w\left(t_{n}\right)=1$, the composition $v=w \circ Z_{1}^{M}$ defines a Vassiliev invariant of degree 1 with $v(K)-v(\theta K)=1$, as desired.

\section{ACKNOWLEDGEMENTS}

I would like to thank Christine Lescop and the referee of my note 11 for their interest in my work and their encouragement to publish this article. Some of the results were part of my Ph.D. thesis [9], which was financially supported by the Deutsche Forschungsgemeinschaft (DFG) through the Graduiertenkolleg Mathematik at the University of Bonn.

\section{REFERENCES}

[1] E. Artin, Theory of braids, Ann. of Math. 48 (1947), 101-126. MR 8:367a

[2] D. Bar-Natan, On the Vassiliev knot invariants, Topology 34 (1995), 423-472. MR 97d:57004 
[3] D. Bar-Natan, Polynomial invariants are polynomial, Math. Res. Lett. 2 (1995), 239-246. MR 96c:57006

[4] J.S. Birman, Braids, links, and mapping class groups, Annals of Mathematics Studies No. 82, Princeton University Press, Princeton, 1974. MR 51:11477

[5] J.S. Birman, X.-S. Lin, Knot polynomials and Vassiliev's invariants, Invent. Math. 111 (1993), 225-270. MR 94d:57010

[6] J. Dean, Many classical knot invariants are not Vassiliev invariants, J. Knot Theory Ramifications 3 (1994), 7-10. MR 94k:57008

[7] P. Dehornoy, Braid groups and left distributive operations, Trans. Amer. Math. Soc. 345 (1994), 115-150. MR 95a:08003

[8] J.L. Dyer, The algebraic braid groups are torsion-free: an algebraic proof, Math. Z. 172 (1980), 157-160. MR 81f:20046

[9] M.Eisermann, Knotengruppen-Darstellungen und Invarianten von endlichem Typ, Ph.D. Dissertation, Bonner Mathematische Schriften No. 327, Bonn 2000. MR 2003g:57014

[10] M. Eisermann, The number of knot group representations is not a Vassiliev invariant, Proc. Amer. Math. Soc. 128 (2000), 1555-1561. MR 2000j:57009

[11] M. Eisermann, Les invariants rationnels de type fini ne distinguent pas les nouds dans $\mathbb{S}^{2} \times \mathbb{S}^{1}$, C. R. Acad. Sci. Paris, Série I, 332 (2001), 51-55. MR 2001m:57019

[12] E. Fadell, J. van Buskirk, The braid groups of $E^{2}$ and $S^{2}$, Duke Math. J. 29 (1962), 243-257. MR 25:4539

[13] E. Fadell, L. Neuwirth, Configuration spaces, Math. Scand. 10 (1962), 111-118. MR 25:4537

[14] R. Fox, L. Neuwirth, The braid groups, Math. Scand. 10 (1962), 119-126. MR 27:742

[15] W.B.R. Lickorish, An introduction to knot theory, Graduate Texts in Mathematics No. 175, Springer-Verlag, New York 1997. MR 98f:57015

[16] J.Lieberum, Invariants de Vassiliev pour les entrelacs dans $S^{3}$ et dans les variétés de dimension trois, Thèse, Prépublication de l'Institut de Recherche Mathématique Avancée no. 1998/30, Strasbourg, 1998. MR 2000g:57025

[17] K. Murasugi, Seifert fibre spaces and braid groups, Proc. London Math. Soc. 44 (1982), 71-84. MR 83f:57007

[18] M.H.A. Newman, On a string problem of Dirac, J. London Math. Soc. 17 (1942), 173-177. MR 4:252g

[19] T. Stanford, The functoriality of Vassiliev-type invariants of links, braids, and knotted graphs, J. Knot Theory Ramifications 3 (1994), 247-262. MR 95h:57011

[20] R. Trapp, Twist sequences and Vassiliev invariants, J. Knot Theory Ramifications 3 (1994), 391-405. MR 95h:57012

[21] S. Willerton, Vassiliev invariants as polynomials, Knot theory (Warsaw 1995), 457-463, Banach Center Publ. No. 42, Polish Acad. Sci., Warsaw 1998. MR 99h:57022

UMPA, École Normale Supérieure de Lyon, 46 allée d'Italie, 69364 Lyon, France

E-mail address: Michael.Eisermann@umpa.ens-lyon.fr

Current address: Institut Fourier, Université Grenoble I, France

E-mail address: Michael.Eisermann@ujf-grenoble.fr

URL: http://www-fourier.ujf-grenoble.fr/ eiserm 